\title{
A METRICAL THEOREM IN DIOPHANTINE APPROXIMATION
}

\author{
WOLFGANG SCHMIDT
}

Introduction. In this paper we prove a sharpening and generalization of the following Theorem of Khintchine (4):

Let $\psi_{1}(q), \ldots, \psi_{n}(q)$ be $n$ non-negative functions of the positive integer $q$ and assume

$$
\psi(q)=\prod_{i=1}^{n} \psi_{i}(q)
$$

is monotonically decreasing. Then the set of inequalities

$$
0 \leqq q \theta_{i}-p_{i}<\psi_{i}(q) \quad(i=1, \ldots, n)
$$

has an infinity of integer solutions $q>0$ and $p_{1}, \ldots, p_{n}$ for almost all or no sets of numbers $\theta_{1}, \ldots, \theta_{n}$, according as $\sum \psi(q)$ diverges or converges.

Actually, Khintchine proved the Theorem with $\left|q \theta_{i}-p_{i}\right|<\psi_{i}(q)$ instead of (1). The first author who used the one-sided inequalities (1) was Cassels (1).

Surprisingly, the following sharpening of the Theorem seems to have escaped attention.

Theorem 1. Make the same assumptions as in Khintchine's Theorem. Let $\epsilon>0$ be arbitrary. Write $N\left(h ; \theta_{1}, \ldots, \theta_{n}\right)$ for the number of solutions of (1) with $1 \leqq q \leqq h$ and put

$$
\begin{aligned}
\Psi(h) & =\sum_{q=1}^{h} \psi(q) \\
\Omega(h) & =\sum_{q=1}^{h} \psi(q) q^{-1} .
\end{aligned}
$$

Then

$$
N\left(h ; e_{1}, \ldots, \theta_{n}\right)=\Psi(h)+O\left(\Psi^{\frac{1}{2}}(h) \Omega^{\frac{1}{2}}(h) \log ^{2+\epsilon} \Psi(h)\right)
$$

for almost all sets $\theta_{1}, \ldots, \theta_{n}$.

Note. In this paper, $\log \alpha$ stands as an abbreviatson for

$$
\left\{\begin{array}{l}
\operatorname{logarithm} \alpha, \text { if } \alpha \geqq e \\
1, \text { if } \alpha<e .
\end{array}\right.
$$

Only $\log (1+1(1 / q-1))$ in $(10)$ means logarithm, in spite of $1+(1 / q-1)<e$.

Next, we generalize Khintchine's Theorem to linear forms. We use the following notation. Throughout this paper, lower case italics denote rational

Received May 4, 1959. 
integers. By $Q, R, \ldots$, we denote lattice points $Q\left(q_{1}, \ldots, q_{m}\right)$ in $R_{m}$. $\theta$ denotes points $\left(\theta_{1}, \ldots, \theta_{m}\right)$ in $R_{m} . \rho Q$, where $\rho$ is real, is the point with co-ordinates $\rho q_{1}, \ldots, \rho q_{m}$, and $Q \theta$ is the scalar product $q_{1} \theta_{1}+\ldots+q_{m} \theta_{m}$. We write $d(Q)$ for the number of common divisors of $q_{1}, \ldots, q_{m}$. Finally, we put $Q \leqq h$ if $q=\max \left(q_{1}, \ldots, q_{m}\right) \leqq h$, and similarly $h<Q$.

Theorem 2. Let $\epsilon>0$ be arbitrary. Let $\psi_{1}(Q), \ldots, \psi_{n}(Q)$ be $n$ bounded non-negative functions. We introduce

$$
\begin{aligned}
& \psi(Q)=\prod_{i=1}^{n} \psi_{i}(Q) \\
& \Psi(h)=\sum_{Q \leqq h} \psi(Q) \\
& \chi(h)=\sum_{Q \leqq h} \psi(Q) d(Q)
\end{aligned}
$$

and write $V\left(h ; \Theta_{1}, \ldots, \Theta_{n}\right)$ for the number of simultaneous solutions $Q \leqq h$, $p_{1}, \ldots, p_{n}$ of the system

$$
0 \leqq Q \Theta_{i}-p_{i}<\psi_{i}(Q) \quad(i=1, \ldots, n) .
$$

Then for almost all n-tuples $\Theta_{1}, \ldots, \Theta_{n}$

$$
N\left(h ; \theta_{1}, \ldots, \theta_{n}\right)=\Psi(h)+O\left(\chi^{\frac{1}{2}}(h) \log ^{3 / 2+\epsilon} \chi(h)\right) .
$$

Note. We need not assume $\psi(Q)$ to be monotonic in any co-ordinate.

This theorem can be interpreted as a generalization of the well-known fact that the points $\left(Q \Theta_{1}, \ldots, Q \Theta_{n}\right)$ are uniformly distributed $\bmod 1$ for almost all $\theta_{1}, \ldots, \Theta_{n}$. (See, for instance, (3, chapter IV).) Indeed, putting $\psi_{i}(Q)=\alpha_{i}$, $\alpha=\prod \alpha_{i}$, we have $\Psi(h)=\alpha h^{m}$ and

$$
\chi(h)=\alpha \sum_{Q \leqq h} d(Q)=\left\{\begin{array}{l}
O(h \log h), \text { if } m=1 \\
O\left(h^{m}\right), \text { if } m>1 .
\end{array}\right.
$$

An interesting special case of Theorem 1 is when $\psi(Q)=\psi(q)$, where $q=\max \left(q_{1}, \ldots, q_{m}\right)$. Then

$$
\begin{aligned}
\chi(h) & =O\left(\sum_{d \leqq h} \sum_{\substack{q_{1} \leqq h \\
d \backslash q_{1}}} \sum_{\substack{q_{2} \leqq q_{1} \\
d \backslash q_{2}}} \ldots \sum_{\substack{q_{m} \leqq q_{1} \\
d \mid q_{m}}} \psi\left(q_{1}\right)\right) \\
& =O\left(\sum_{d \leqq h} \sum_{\substack{q_{1} \leqq h \\
d \mid q_{1}}} \psi\left(q_{1}\right)\left(\frac{q_{1}}{d}\right)^{m-1}\right) .
\end{aligned}
$$

Thus we have

$$
\chi(h)=O(\Psi(h))
$$

if $m \geqq 3$, or if $m=2$ and $q \psi(q)$ is monotonically decreasing, because in the latter case

$$
\sum_{\substack{q_{1} \leqq h \\ d \mid q_{1}}} \psi\left(q_{1}\right) q_{1} \leqq d^{-1} \Psi(h)
$$


For example, if $\psi_{i}(Q)=\psi_{i}(q)=q^{-m / n}, \psi(Q)=q^{-m}, \Psi(h)=m \log h+$ $O(1)$, then for almost all $\theta_{1}, \ldots, \theta_{m}$

$$
N\left(h ; \theta_{1}, \ldots, \theta_{m}\right)=m \log h+O\left(\log ^{\frac{1}{2}} h \log \log ^{\alpha+\epsilon} h\right),
$$

where we may take $\alpha=2$ for $m=1$, according to Theorem 1 , and $\alpha=3 / 2$ for $m>1$, according to Theorem 2 .

For the proof we have to modify the standard proof of Khintchine's Theorem and use some ideas of (2). The new idea in Theorem 1 is to use fractions $p / q$ with g.c.d. $(p, q) \leqq k$ where $k$ is specified later, instead of $p / q$ with g.c.d. $(p, q)=1$, as employed in $(1 ; 3 ; 4)$. Theorems 1 and 2 should be compared with similar results I proved recently in the geometry of numbers (5).

We give a detailed proof of Theorem 1 only. For convergent sums $\sum \psi(q)$ Theorem 1 follows from Khintchine's Theorem. Hence in $\S 1$ to 4 , which deal with Theorem 1 , we assume without explicit mention that $\psi(q)$ is a non-negative, monotonically decreasing function with divergent sum $\sum \psi(q)$. $\Psi(h)$ and $\Omega(h)$ are defined by (2) and (3). The author is much indebted to the referee who discovered a mistake in the original draft and made valuable suggestions.

1. On certain intervals. Let $\omega(h), h \geqq 1$, be a monotonically increasing integral-valued function which tends to infinity. We write $\omega(0)=0$ and define $S^{\prime}$ to be the set consisting of 0 and of all integers $h>0$ such that $\omega(h-1)<\omega(h)$. We define $S^{\prime \prime}$ to be the set of integers $h \geqq 0$ having $\omega(h)<\omega(h+1)$. Finally, $S$ is the set of values of $\omega(h), h \geqq 0$.

Next, we define for fixed $t>0$ intervals of order $t$ to be the half-open intervals

$$
\left(u 2^{t}+v_{1},(u+1) 2^{t}+v_{2}\right],
$$

where $u, v_{1}, v_{2}$ are non-negative integers such that $v_{1}<2^{t}$ and $v_{1}, v_{2}$ are the smallest non-negative integers satisfying $u 2^{t}+v_{1} \in S,(u+1) 2^{t}+v_{2} \in S$. (It is possible, of course, that for given $u, t$ there exists no such $v_{1}$.) The intervals of order $t$ cover the positive axis exactly once.

Lemma 1. Every interval $(0, x], x \in S$, can be expressed as union of intervals $\cup I_{i}$ of the type described above, where no two of the intervals $I_{i}$ are of the same order.

Proof. Write $x$ in the binary scale,

$$
x=\sum_{i=0}^{w} t_{i} 2^{i}
$$

where $t_{i}$ equals 0 or 1 , but $t_{w}=1$. There exists an interval $\left(0, j_{1}\right]$ of order $w$ with $j_{1} \leqq x$. If $j_{1}=x$, then we are through. If not, and if

$$
j_{1}=\sum_{i=0}^{w} t_{i}^{(2)} 2^{i}
$$


then $t_{w}{ }^{(2)}=t_{w}=1$ and there exists a largest integer $w_{2}$ having

$$
t_{w 2}^{(2)}<t_{w 2}
$$

Hence there exists an interval $\left(j_{1}, j_{2}\right]$ of order $w_{2}, j_{2} \leqq x$. If $j_{2}=x$, then $(0, x]=\left(0, j_{1}\right] \cup\left(j_{1}, j_{2}\right]$. Otherwise, if

$$
j_{2}=\sum_{i=0}^{w} t_{i}^{(3)} 2^{i}, \quad t_{w}^{(3)}=t_{w}=1, \ldots, t_{w 2}^{(3)}=t_{w_{2}}=1,
$$

then there exists a largest $w_{3}, w_{3}<w_{2}$, having

$$
t_{w_{3}}^{(3)}<t_{w_{3}} \text {. }
$$

We proceed as before. Since $j_{1}<j_{2}<\ldots$, we finally arrive at $j_{f}=x$ and $(0, x]=\left(0, j_{1}\right] \cup \ldots \cup\left(j_{f-1}, j_{f}\right]$. The orders of the intervals are $w>w_{2}$ $>\ldots>w_{f}>0$.

2. Sums involving a function $\phi(k, q)$. Let $k, q$ be positive and write $\phi(k, q)$ for the number of integers $x, 0 \leqq x<q$, so that g.c.d. $(x, q) \leqq k$.

LEMMA 2.

$$
\sum_{q=1}^{v} \phi(k, q) q^{-1}=v+O\left(v k^{-1}+\log v \log k\right) .
$$

Note. Here and throughout the paper, the inequality indicated by the $O$-symbol holds for all values of all variables involved.

Proof. Clearly,

$$
\phi(k, q)=\sum_{\substack{w \mid q \\ w \leqq k}} \phi\left(\frac{q}{w}\right),
$$

where $\phi(x)$ is the Euler $\phi$-function. Using the well-known relation

$$
\phi(x)=x \sum_{y \mid x} \mu(y) y^{-1}
$$

we obtain

$$
\begin{aligned}
\sum_{q=1}^{v} \phi(k, q) q^{-1} & \\
= & \sum_{q=1}^{v} q^{-1} \sum_{\substack{w \mid q \\
w \leqq k}} q w^{-1} \sum_{y \mid q w^{-1}} \mu(y) y^{-1} \\
& =\sum_{w=1}^{\min (k, v)} w^{-1} \sum_{y=1}^{[(v / w)]} \mu(y) y^{-1} \sum_{q=1}^{[(v / y w)]} 1,
\end{aligned}
$$

where $[\alpha]$ is the integral part of $\alpha$. Thus 


$$
\begin{aligned}
\sum_{q=1}^{v} \phi & (k, q) q^{-1} \\
& =v \sum_{w=1}^{\min (k, v)} w^{-2} \sum_{y=1}^{[(v / w)]} \mu(y) y^{-2}+O(\log v \log k) \\
& =v \sum_{w=1}^{\min (k, v)} w^{-2} \xi(2)^{-1}+O\left(\sum_{w=1}^{\min (k, v)} w^{-1}\right)+O(\log v \log k) \\
& =v+O\left(v k^{-1}+\log v \log k\right) .
\end{aligned}
$$

Lemma 3.

$$
\sum_{q=1}^{v} \psi(q) \phi(k, q) q^{-1}=\Psi(v)+O\left(\Psi(v) k^{-1}+\Omega(v) \log k\right) .
$$

Proof. Put $\Pi(k, 0)=0$ and

$$
\Pi(k, r)=\sum_{q=1}^{r} \phi(k, q) q^{-1}
$$

for $r \geqq 1$. Lemma 2 yields

$$
\Pi(k, r)=r+O\left(r k^{-1}+\log r \log k\right) .
$$

Using partial summation we obtain

$$
\begin{aligned}
\sum_{q=1}^{v} \psi(q) & \phi(k, q) q^{-1} \\
& =\sum_{q=1}^{v} \psi(q)(\Pi(k, q)-\Pi(k, q-1)) \\
& =\sum_{q=1}^{v-1} \Pi(k, q)(\psi(q)-\psi(q+1))+\Pi(k, v) \psi(v) \\
& =\sum_{q=1}^{v-1} q(\psi(q)-\psi(q+1))+v \psi(v)+R(k, v) \\
& =\Psi(v)+R(k, v),
\end{aligned}
$$

where, according to (7),

$$
\begin{aligned}
& R(k, v) \\
& =O\left(\sum_{q=1}^{v-1}\left(q k^{-1}+\log q \log k\right)(\psi(q)-\psi(q+1))\right. \\
& \quad+O\left(v k^{-1}+\log v \log k\right) \psi(v) \\
& =O\left(\Psi(v) k^{-1}+\log k \sum_{q=2}^{v} \psi(q)(\log q-\log (q-1))+\log k \psi(1)\right) .
\end{aligned}
$$


Now

$$
\begin{aligned}
\sum_{q=2}^{v} \psi(q) & (\log q-\log (q-1)) \\
= & O\left(\sum_{q=2}^{v} \psi(q) \log \left(1+\frac{1}{q-1}\right)\right) \\
= & O(\Omega(v)) .
\end{aligned}
$$

Lemma 3 is a consequence of (8), (9), and (10).

3. Bounds for certain integrals. We introduce the following functions and integrals.

$$
\begin{aligned}
\beta(q, \theta) & =\left\{\begin{array}{l}
1, \text { if } 0 \leqq \theta<\psi(q) \\
0 \text { otherwise, }
\end{array}\right. \\
\gamma(q, \theta) & =\sum_{p} \beta(q, q \theta-p), \\
\gamma(k, q, \theta) & =\sum_{\substack{p \\
\text { g.c.d. }(p, q) \leqq k}} \beta(q, q \theta-p), \\
I(q) & =\int_{0}^{1} \gamma(q, \theta) d \theta, \\
I(k ; q) & =\int_{0}^{1} \gamma(k, q, \theta) d \theta, \\
I(k ; q, r) & =\int_{0}^{1} \gamma(k, q, \theta) \gamma(k, r, \theta) d \theta, \\
\Psi(u, v) & =\sum_{u+1}^{v} \psi(q) .
\end{aligned}
$$

We observe

$$
N(v, \theta)=\sum_{q=1}^{v} \gamma(q, \theta)
$$

and put

$$
N(k ; u, v ; \theta)=\sum_{q=u+1}^{v} \gamma(k, q, \theta)
$$

LEMMA 4.

$$
\begin{aligned}
I(q) & =\psi(q) ; \quad I(k ; q)=\psi(q) \phi(k, q) q^{-1} \\
\mathrm{I}(k ; q, r) & \leqq \psi(q) \psi(r)+A(k ; q, r) \psi(q) q^{-1},
\end{aligned}
$$

where $A(k ; q, r)$ is the number of solutions $p, s$ of 


$$
q s-r p=0
$$

$0 \leqq p<q$

having

$$
\text { g.c.d. }(p, q) \leqq k, \quad \text { g.c.d. }(s, r) \leqq k .
$$

Proof. $I(q)=\psi(q)$ is rather trivial, while the second half of (11) follows from

$$
\begin{aligned}
I(k, q) & =\sum_{\substack{p \\
\text { g.c.... }(p, q) \leqq k}} \int_{0}^{1} \beta(q, \theta q-p) d \theta \\
& =\phi(k, q) q^{-1} \int_{-\infty}^{\infty} \beta(q, \theta) d \theta .
\end{aligned}
$$

As for $I(k ; q, r)$, we have

$$
I(k ; q, r)=\sum_{\substack{p, \text { g.c.d. }(p, q) \leqq k \\ s, g . c . \text {.d. }(s, r) \leqq k}} \int_{0}^{1} \beta(q, \theta q-p) \beta(r, \theta r-s) d \theta .
$$

We split this sum into two parts,

$$
I(k ; q, r)=I_{0}(k ; q, r)+I_{1}(k ; q, r),
$$

where $I_{0}$ consists of the terms with $q s-r p \neq 0$.

$$
\begin{aligned}
I_{0}(k ; q, r) & \leqq \sum_{\substack{p, s, q s-r p \neq 0}} \int_{0}^{1} \beta(q, \theta q-p) \beta(r, \theta r-s) d \theta \\
& =\sum_{\substack{p, s \\
q s-r \neq \neq 0}} \int_{-(p / q)}^{1-(p / q)} \beta\left(q, q \theta^{\prime}\right) \beta\left(r, r \theta^{\prime}-\frac{q s-r p}{q}\right) d \theta^{\prime} .
\end{aligned}
$$

To find an estimate for this sum, write $q=q^{\prime} d, r=r^{\prime} d, q s-r p=h d$, where $d=$ g.c.d. $(q, r) \cdot$ For given $h, p$ is determined modulo $q^{\prime}$. Hence

$$
\begin{aligned}
& I_{0}(k ; q, r) \\
& \quad \leqq d \sum_{h \neq 0} \int_{-\infty}^{\infty} \beta\left(q, q \theta^{\prime}\right) \beta\left(r, r \theta^{\prime}-\frac{h d}{q}\right) d \theta^{\prime} \\
& \quad \leqq d \int_{-\infty}^{\infty} \int_{-\infty}^{\infty} \beta\left(q, q \theta^{\prime}\right) \beta\left(r, r \theta^{\prime}-\lambda d q^{-1}\right) d \theta^{\prime} d \lambda \\
& \quad=\psi(q) \psi(r) .
\end{aligned}
$$

In changing from the summation over $h$ to the continuous parameter $\lambda$ we used the fact that the function

$$
\int_{-\infty}^{\infty} \beta\left(q, q \theta^{\prime}\right) \beta\left(r, r \theta^{\prime}-\lambda d q^{-1}\right) d \theta^{\prime}
$$

is monotonically decreasing in $\lambda$ when $\lambda \geqq 0$, and monotonically increasing when $\lambda \leqq 0$. 
To prove Lemma 4 it remains to give an upper bound for $I_{1}(k ; q, r)$. In analogy to (14), we find

$$
\begin{aligned}
I_{1}(k ; q, r) & =\sum_{\substack{p, \text { g. .e.d. }(p, q) \leqq k \\
\text { s,g.c.d. }(s, r) \leq k \\
q s-r p=0}} \int_{-(p / q)}^{1-(p / q)} \beta\left(q, q \theta^{\prime}\right) \beta\left(r, r \theta^{\prime}\right) d \theta^{\prime} \\
& \leqq A(k ; q, r) \psi(q) q^{-1} .
\end{aligned}
$$

Lemma 5.

$$
\begin{aligned}
& \int_{0}^{1} N(v, \theta) d \theta=\Psi(v) \\
& \int_{0}^{1} N(k ; u, v ; \theta) d \theta=\sum_{q=u+1}^{v} \psi(q) \phi(k, q) q^{-1} \\
& \int_{0}^{1} N^{2}(k ; u, v ; \theta) d \theta \leqq \Psi^{2}(u, v)+2 \sum_{q=u+1}^{v} \psi(q) d_{k}(q),
\end{aligned}
$$

where $d_{k}(q)$ is the number of divisors of $q$ not exceeding $k$.

Proof. The first two assertions follow from (11). As an immediate consequence of (12) we have

$$
\int_{0}^{1} N^{2}(k ; u, v ; \theta) d \theta \leqq \Psi^{2}(u, v)+2 \sum_{u<r \leqq q \leqq v} A(k ; q, r) \psi(q) q^{-1} .
$$

Now

$$
\sum_{r=1}^{q} A(k ; q, r)
$$

is equal to the number of solutions $r, p, s$ of

$$
\begin{gathered}
q s-r p=0, \quad 0 \leqq p<q, \quad 1 \leqq r \leqq q \\
\text { g.c.d. }(p, q) \leqq k, \quad \text { g.c.d. }(s, r) \leqq k .
\end{gathered}
$$

Define $a, b$ by

$$
\frac{a}{b}=\frac{p}{q}=\frac{s}{r}, \quad \text { g.c.d. }(a, b)=1 .
$$

Then $b / q$ and g.c.d. $(p, q) \leqq k$ implies $q b^{-1} \leqq k$. Thus the number of possible choices for $b$ is $d_{k}(q)$. Furthermore, there are $\phi(b) \leqq b$ possibilities for $a$ and $q b^{-1}$ possibilities for $r$, once $b$ is given. Hence

$$
\sum_{r=1}^{q} A(k ; q, r) \leqq q d_{k}(q)
$$

and

$$
\sum_{u<r \leqq q \leqq v} A(k ; q, r) \psi(q) q^{-1} \leqq \sum_{q=u+1}^{v} \psi(q) d_{k}(q)
$$


4. Proof of Theorem $1(n=1)$. Write $\omega(h)=[\Psi(h) \Omega(h)]$ and define $S, S^{\prime}, S^{\prime \prime}$ as in $\S 1$. Let $L_{s}$ be the set of all pairs $(u, v), u \in S^{\prime}, v \in S^{\prime}$, so that $(\omega(u), \omega(v)]$ is an interval of any order $t$ with respect to $\omega$ (see $\S 1$ ), and $\omega(v) \leqq 2^{s}$. From now on, the numbers $k, s$ are always connected by the relation

$$
k=2^{s} \text {. }
$$

From here on, we make heavy use of the methods developed in (2). Write $h^{*}=h^{*}(s)$ for the largest integer $h^{*}$ having $\omega\left(h^{*}\right) \leqq 2^{s}$.

LEMMA 6.

$$
\begin{gathered}
0 \leqq \int_{0}^{1}\left(N\left(h^{*}, \theta\right)-N\left(k ; 0, h^{*} ; \theta\right)\right) d \theta=O\left(s 2^{s / 2}\right) \\
\sum_{(u, v) \in L_{s}} \int_{0}^{1}(N(k ; u, v ; \theta)-\Psi(u, v))^{2} d \theta=O\left(s^{2} 2^{s}\right) .
\end{gathered}
$$

Proof. The first two equations of Lemma 5 give

$$
\begin{aligned}
\int_{0}^{1}\left(N\left(h^{*}, \theta\right)\right. & \left.-N\left(k ; 0, h^{*}, \theta\right)\right) d \theta \\
= & \Psi\left(h^{*}\right)-\sum_{q=1}^{h^{*}} \psi(q) \phi(k, q) q^{-1} \\
= & O\left(\Psi\left(h^{*}\right) k^{-1}+\Omega\left(h^{*}\right) \log k\right.
\end{aligned}
$$

according to Lemma 3 . Since

$$
\Omega\left(h^{*}\right)=O\left(2^{\frac{1}{2} s}\right),
$$

(16) follows.

Using Lemma 5 again we see that a single integral in (17) does not exceed

$$
2 \sum_{q=u+1}^{v} \psi(q) d_{k}(q)+2 \Psi(u, v)\left(\Psi(u, v)-\sum_{q=u+1}^{v} \psi(q) \phi(k, q) q^{-1}\right) .
$$

We first take the sum over those pairs $(u, v) \in L_{s}$ where $(\omega(u), \omega(v)]$ is an interval of fixed order $t$. Since intervals of order $t$ cover the positive axis exactly once, we obtain the upper bound

$$
2 \sum_{q=1}^{h^{*}} \psi(q) d_{k}(q)+2 \Psi\left(h^{*}\right)\left(\Psi\left(h^{*}\right)-\sum_{q=1}^{h^{*}} \psi(q) \phi(k, q) q^{-1}\right) .
$$

We observe

$$
\sum_{q=1}^{h^{*}} \psi(q) d_{k}(q) \leqq 2^{s} \sum_{t=1}^{k} t^{-1}=O\left(2^{s} \log k\right)
$$

and using Lemma 3 we find the upper bound

$$
O\left(2^{s} \log k\right)+O\left(\Psi^{2}\left(h^{*}\right) k^{-1}+\Psi\left(h^{*}\right) \Omega\left(h^{*}\right) \log k\right)=O\left(\mathrm{~s} 2^{s}\right) .
$$

Summing over $t$ and observing $t \leqq s$ we obtain (17). 
Lemma 7. There is a sequence of subsets $\sigma_{1}, \sigma_{2}, \ldots$ of the unit-interval with measures

$$
\mu_{s}=\int_{\sigma_{s}} d \theta=O\left(s^{-1-\epsilon}\right)
$$

such that

$$
N(h, \theta)=\Psi(h)+O\left(2^{s / 2} s^{2+\epsilon}\right)
$$

for any $h$ with $\omega(h) \leqq 2^{s}, h \in S^{\prime}$, and any $\theta$ in $0 \leqq \theta<1$, but not in $\sigma_{s}$.

Proof. We define $\sigma_{s}$ to be the set of all $\theta$ in $0 \leqq \theta<1$, for which not both of the following two inequalities hold:

$$
\begin{gathered}
0 \leqq N\left(h^{*}, \theta\right)-N\left(k ; 0, h^{*} ; \theta\right) \leqq s^{2+\epsilon} 2^{\frac{1}{2} s} \\
\sum_{(u, v) \epsilon L_{s}}(N(k ; u, v ; \theta)-\Psi(u, v))^{2} \leqq s^{3+\epsilon} 2^{s} .
\end{gathered}
$$

As a consequence of Lemma 6 ,

$$
\mu_{s}=O\left(s^{-1-\epsilon}\right) \text {. }
$$

If $h \leqq h^{*}, h \in S^{\prime}$, then the interval $(0, \omega(h)]$ is the union of at most $s$ intervals $(\omega(u), \omega(v)]$, where $(u, v) \in L_{s}$.

$$
N(k ; 0, h ; \theta)-\Psi(h)=\sum(N(k ; u, v ; \theta)-\Psi(u, v)),
$$

where the sum is over at most $s$ pairs $(u, v) \in L_{s}$. This fact, together with (19) and Cauchy's inequality yields for $0 \leqq \theta<1, \theta \notin \sigma_{s}$,

$$
(N(k ; 0, h ; \theta)-\Psi(h))^{2} \leqq s^{4+\epsilon} 2^{s} .
$$

The last equation together with (18) gives Lemma 7.

Proof of Theorem $1(n=1)$. Since $\sum s^{-1-\epsilon}$ is convergent, there exists for almost all $\theta, 0 \leqq \theta<1$, an $s_{0}=s_{0}(\theta)$ such that $\theta \notin \sigma_{s}$ for $s \geqq s_{0}$. Assume $\theta$ has such an $s_{0}(\theta)$ and assume $h$ to be so large that $\omega(h) \geqq 2^{s_{0}}$. Choose $s$ so that $2^{s-1} \leqq \omega(h)<2^{s}$.

Suppose $h \in S^{\prime}$. Then we have with Lemma 7

$$
\begin{aligned}
N(h, \theta) & =\Psi(h)+O\left(2^{\frac{1}{2} s} s^{2+\epsilon}\right) \\
& =\Psi(h)+O\left(\Psi^{\frac{1}{2}}(h) \Omega^{\frac{1}{2}}(h) \log ^{2+\epsilon} \Psi(h)\right) .
\end{aligned}
$$

Hence Theorem 1 holds for $h \in S^{\prime}$. By the same argument we can prove the Theorem for $h \in S^{\prime \prime}$.

To any $h$ there exist $h^{\prime}, h^{\prime \prime}$ with $h^{\prime} \in S^{\prime}, h^{\prime \prime} \in S^{\prime \prime}$ and

$$
\begin{aligned}
& \omega\left(h^{\prime}\right)=\omega(h)=\omega\left(h^{\prime \prime}\right) . \\
& \left|\Psi(h) \Omega(h)-\Psi\left(h^{\prime}\right) \Omega\left(h^{\prime}\right)\right| \leqq 1 .
\end{aligned}
$$

Then

$$
\left|\Psi(h)-\Psi\left(h^{\prime}\right)\right| \leqq \Omega(h)^{-1} \leqq \Omega(1)^{-1}=\psi(1)^{-1},
$$


and similarly for $\Psi\left(h^{\prime \prime}\right)$. Since

$$
N\left(h^{\prime}, \theta\right) \leqq N(h, \theta) \leqq N\left(h^{\prime \prime}, \theta\right),
$$

the case $n=1$ of Theorem 1 follows.

\section{The case $n \geqq 2$. Using}

$$
\begin{aligned}
v-\sum_{q=1}^{v} & \phi^{n}(k, q) q^{-n} \\
& =\sum_{q=1}^{v}\left(q^{n}-\phi^{n}(k, q)\right) q^{-n} \\
& \leqq n \sum_{q=1}^{v}(q-\phi(k, q)) q^{n-1} q^{-n} \\
& =n\left(v-\sum_{q=1}^{v} \phi(k, q) q^{-1}\right)
\end{aligned}
$$

we easily generalize Lemmas 2,3 to

$$
\begin{gathered}
\sum_{q=1}^{v} \phi^{n}(k, q) q^{-n}=v+O\left(v k^{-1}+\log k \log v\right) \\
\sum_{q=1}^{v} \psi(q) \phi^{n}(k, q) q^{-n}=\Psi(v)+O\left(\Psi(v) k^{-1}+\Omega(v) \log k\right) .
\end{gathered}
$$

In analogy to $\beta(q, \theta)$ of $\S 3$ we define $\beta\left(q, \theta_{1}, \ldots, \theta_{n}\right)$ to be the characteristic function of the rectangle

$$
0 \leqq \theta_{i}<\psi_{i}(q) \quad(i=1, \ldots, n)
$$

and put

$$
\begin{aligned}
& \gamma\left(q, \theta_{1}, \ldots, \theta_{n}\right)=\sum_{p_{1}, \ldots, p_{t *}} \beta\left(q, q \theta_{1}-p_{1}, \ldots, q \theta_{n}-p_{n}\right) \\
& \gamma\left(k ; q, \theta_{1}, \ldots, \theta_{n}\right)=\sum_{\substack{p_{i}, \text { g. c..d. }\left(p_{i}, q\right) \leqq k \\
i=1, \ldots, n}} \beta\left(q, q \theta_{1}-p_{1}, \ldots, q \theta_{n}-p_{n}\right) .
\end{aligned}
$$

$I(q), I(k, q), I(k ; q, r)$ are now $n$-dimensional integrals. To find an upper bound for

$$
\begin{aligned}
& I(k ; q, r)=\sum_{\substack{p_{i} \text {,g.c.d. }\left(p_{i}, q\right) \leqq k \\
s_{i} \text {.g.e.c.t. }\left(i_{i}, r\right) \leqq k \\
i=1, \ldots, n}} \int_{0}^{1} \ldots \int_{0}^{1} \beta\left(q, q \theta_{1}-p_{1}, \ldots,\right) \\
& \beta\left(r, r \theta_{1}-s_{1}, \ldots,\right) d \theta_{1} \ldots d \theta_{n},
\end{aligned}
$$

we split this sum into $n+1$ parts,

$$
I(k ; q, r)=I_{0}+\ldots+I_{n}
$$

where $I_{j}$ consists of the terms with exactly $j$ indices $i_{1}, \ldots, i_{\text {, having }}$ $q s_{i}-r p_{i}=0$. We find 


$$
I_{0}(k ; q, r) \leqq \psi(q) \psi(r)
$$

and

$$
\begin{aligned}
I_{j}(k ; q, r) & \leqq c^{(j)} A^{j}(k ; q, r) \psi(q) q^{-j} \\
& \leqq c^{(j)} A(k ; q, r) \psi(q) q^{-1} .
\end{aligned}
$$

There are no other modifications of any depth.

6. On the proof of Theorem 2. For simplicity assume $n=1$. We put

$$
\beta(Q, \theta)=\left\{\begin{array}{l}
1, \text { if } 0 \leqq \theta<\psi(Q) \\
0 \text { otherwise }
\end{array}\right.
$$

and define $\gamma(Q, \theta), I(Q)$ in an obvious way. Further

$$
\begin{aligned}
& I(Q, R)=\int_{0}^{1} \gamma(Q, \theta) \gamma(R, \theta) d \theta, \\
& \Psi(u, v)=\sum_{u<Q \leqq v} \psi(Q) .
\end{aligned}
$$

We observe

$$
N(v, \theta)=\sum_{Q \leqq v} \gamma(Q, \theta)
$$

and put

$$
N(u, v, \theta)=\sum_{u<Q \leqq v} \gamma(Q, \theta) .
$$

We do not need the parameter $k$ now, which was essential in Theorem 1 . Lemma 4 now reads

Lemma $4 a$.

$$
\begin{aligned}
I(Q) & =\psi(Q) \\
I(Q, R) & =\psi(Q) \psi(R),
\end{aligned}
$$

if $Q, R$ are linearly independent (there exists no $\rho$ having $Q=\rho R$ ).

$$
I(Q, R) \leqq \psi(Q) \psi(R)+c A\left(q_{1}, r_{1}\right) \psi(Q) q_{1}^{-1},
$$

if $Q, R$ are linearly dependent. Here $q_{1}, r_{1}$ are the first co-ordinates of $Q, R$ and $A\left(q_{1}, r_{1}\right)$ is the number of solutions $p, s$ of

$$
q_{1} s-r_{1} p=0 \quad 0 \leqq p<q .
$$

(20) and (21) are proved like (11), while the proof of (22) is like the one given for (12). Lemma 5 becomes

Lemma $5 a$.

$$
\begin{aligned}
& \int_{0}^{1} N(u, v, \theta) d \theta=\sum_{u<Q \leqq v} \psi(Q)=\Psi(u, v) \\
& \int_{0}^{1} N^{2}(u, v, \theta) d \theta \leqq \Psi^{2}(u, v)+c \sum_{u<Q \leqq v} \psi(Q) d(Q) .
\end{aligned}
$$


All the other changes in the proof are obvious, except perhaps the definition of $\omega(h)$, namely $\omega(h)=[\chi(h)]$.

\section{REFERENCES}

1. J. W. S. Cassels, Some metrical theorems in diophantine approximation I, Proc. Camb. Phil. Soc., 46 (1950), 209-218.

2. - Some metrical theorems in diophantine approximation III, Proc. Camb. Phil. Soc., 46 (1950), 219-225.

3. - An introduction to diophantine approximation, Cambridge Tracts, 45 (1957).

4. A. Khintchine, Zur metrischen Theorie der diophantischen Approximationen, Math. Z., 24 (1926), 706-714.

5. W. Schmidt, A metrical theorem in geometry of numbers, Trans. Amer. Math. Soc., OO (1960), 000-000.

Montana State University 